\title{
THE RELATIONSHIP BETWEEN LEVELS OF PHYSICAL ACTIVITY AND QUALITY OF LIFE AMONG STUDENTS OF THE UNIVERSITY OF THE THIRD AGE
}

\author{
Justyna Krzepota', Elżbieta Biernat ${ }^{2}$, Beata Florkiewicz ${ }^{1}$ \\ 'Department of Physical Culture and Health Promotion, University of Szczecin, Szczecin, Poland \\ ${ }^{2}$ Department of Tourism, Collegium of World Economy, Warsaw School of Economics, Warsaw, Poland
}

\begin{abstract}
SUMMARY
Aim: The assessment of quality of life (QoL) among elderly people is of great importance when preparing health care programmes for this social group. Many researchers indicate that poor health is less frequently observed in senior citizens who display levels of physical activity (PA) prescribed by WHO. The aim of the study was to investigate the relationship between the level of PA among students of the University of the Third Age (U3A) and their self-assessment concerning their QoL.

Methods: The research sample consisted of 131 students from three U3A in West Pomeranian Voivodeship in Poland. PA was assessed with the aid of a short Polish version of the International Physical Activity Questionnaire (IPAQ). The assessment of QoL was conducted with the use of a Polish version of the WHO Quality of Life - BREF instrument (WHOQOL-BREF).

Results: The present study has shown that highly active U3A students declare high QoL in the psychological and social domains more often than other respondents.

Conclusion: The level of PA recommended by WHO has a positive impact on the perceived QoL. Possible differences are visible mostly in the assessment of different domains of life in relation to the presented levels of PA.
\end{abstract}

Key words: physical activity, quality of life, U3A, older adults, aging

Address for correspondence: J. Krzepota, Department of Physical Culture and Health Promotion, University of Szczecin, al. Piastów 40b, blok 6, 71-065 Szczecin, Poland. E-mail: justyna.krzepota@univ.szczecin.pl

\section{INTRODUCTION}

The assessment of quality of life among students of the University of the Third Age and searching for factors affecting the good self-rated health are of great importance when preparing health care programmes for seniors (1-3). Previous research has shown that a lower index of life satisfaction among senior citizens may lead to an increased risk of mortality (4) and a higher number of suicides (5). On the other hand, greater satisfaction and happiness can be connected with a longer life $(6,7)$. Nevertheless, there is no unambiguous stand as to how the assessment of quality of life influences the predictability of illnesses and mortality (8). The factors influencing the quality of life and the risk of committing suicide are being searched (9). It is also indicated that the motivation for improving the quality of life differs in men and women (10). Moreover, positive effects of exercise on mood in older adults have been observed (11). However, Menec (12) pointed out that different types of daily activities can have different benefits for people.

Researchers indicate that adults who display levels of physical activity recommended by WHO had better scores on some dimensions of HRQOL than the inactive ones (13). It has been observed that people suffering from cancer, who adhere to the WHO recommendations for physical activity, tend to feel bet- ter about their quality of life than healthy people not adhering to these recommendations (14). Moreover, research shows that systematic physical exercise reduces the risk of death (up to $35 \%$ ) in comparison with sedentary lifestyle, thus, researchers pointed out that in order to decrease mortality in older adults, the energy expenditure through regular exercise should be at least $1000 \mathrm{kcal}$ per week (15). Researches stated that the systematic physical exercise is positively correlated with independence, aerobic resistance and quality of life of elderly women (16), and negatively correlated with cognitive decline, depression and high perceived stress levels (17).

Some authors (18) claim that there is no precise exercise recommendation (frequency, intensity and duration) for mental health promotion. Chen et al. (19) recommend that in order to improve mental well-being elderly people should focus on higher intensity activity instead of its frequency and duration, but other research (18) suggests that moderate level of physical activity could improve mental well-being. Lee et al. (20) suggest that instead of prescribing activities of $\geq 3$ METs for all people, the individual fitness level should be taken into account in order to reduce the prevalence of chronic diseases. In turn, the latest reports (21) indicate that in order to improve public health and reduce the prevalence of chronic diseases, it is necessary to promote increasing physical activity with high intensity. 
However, different methodological approaches used in the research (and consequently inconsistent results) make it impossible to take an unequivocal stand on the matter, thus the problem needs further investigation $(22,23)$.

The aim of the study is to investigate the relationship between the level of physical activity (high, moderate and low) among students of the University of the Third Age (U3A), and their self-assessment concerning their general quality of life and health condition as well as the quality of life in four domains: physical, psychological, social, and environmental.

\section{MATERIALS AND METHODS}

The research sample consisted of 131 students (22 men and 109 women) from three Universities of the Third Age in West Pomeranian Voivodeship in Poland. In line with the requirements of one of the applied tools (International Physical Activity Questionnaire - IPAQ), the age of respondents was not higher than 69. The average age was $61.4 \pm 4.97$ years, $38.2 \%$ of participants had higher education, $55.0 \%$ completed a secondary school, and $6.1 \%$ a primary school.

The physical activity of respondents was assessed with the aid of a short version of the International Physical Activity Questionnaire (IPAQ), adapted to Polish conditions (24). The assessment of quality of life was conducted with the use of a Polish version of the WHO Quality of Life - BREF instrument, adapted by Wołowicka et al. (25).

\section{International Physical Activity Questionnaire (IPAQ) - Short Version}

IPAQ was used to gather information on the frequency and duration of physical exercise (intense, moderate, and walking) undertaken by the respondents in the previous week (at work, at home, in their spare time, during transportation etc.). Intense physical exercise was defined as exercise causing very fast breathing and heartbeat (i.e. lifting heavy objects, digging, aerobics, fast running, fast bicycle riding). Moderate physical exercise was defined as activity leading to slightly faster breathing and heartbeat (i.e. carrying lighter objects, riding a bicycle at a regular pace, playing volleyball, very fast walking). Walking connected with transportation, shopping and strolling were subjects to analysis.

Each type of physical exercise was defined in MET minutes per week, that is by multiplying the metabolic equivalent assigned to a given exercise (intense: $8 \mathrm{MET}$, moderate: $4 \mathrm{MET}$, walking: 3.3 MET) by the number of days per week it was undertaken and the average duration in minutes per day. According to the IPAQ guidelines, three levels of activity were distinguished. High: 3 or more days of intense physical exercise, altogether at least 1,500 MET min/week; 7 or more days of mixed physical activity (walking, moderate and intense exercise) exceeding the value of 3,000 MET min/week. Moderate: 3 or more days of intense physical exercise, not less than 20 minutes a day; 5 or more days of moderate physical exercise or walking at least 30 minutes a day; 5 or more days of mixed physical activity (walking, moderate and intense exercise) exceeding the value of $600 \mathrm{MET}$ min/week. Low: this group includes people who are not physically active or do not meet the requirements of the high and moderate levels.

\section{WHO Quality of Life - BREF Questionnaire}

The WHOQOL-BREF questionnaire consists of 26 questions. The first two questions were analysed separately. They pertained to the general self-perception of the respondents' quality of life and health. The remaining 24 questions assessed four aspects of the quality of life (physical - 7 questions, psychological - 6 questions, social -3 questions, and environmental -8 questions). The respondents were asked to mark their answers using a five level rating scale (from 1 to 5 points). The quality of life in respective domains was expressed as a mean value, calculated according to the key and guidelines provided by Wołowicka et al. (25). The higher the score, the better the quality of life.

\section{Statistics}

The differences between mean values of the analysed WHOQOL-BREF domains (physical, psychological, social, and environmental), depending on the level of physical activity (high, moderate and low) of the respondents, were assessed with the aid of one-way ANOVA. Normal distribution was analysed using Shapiro-Wilk test, and Levene's test was applied to assess the equality of variances. All analyses were conducted with the use of a statistics software package STATISTICA 9. 0 PL. The significance of factors included in the analysis was assessed using Chi-squared test, and the level of significance adopted was $\mathrm{p}<0.05$.

\section{RESULTS}

The level of physical activity among students of the Univeristy of the Third Age was assessed using a short version of IPAQ. WHO recommendations are followed by $61.1 \%$ of all seniors, $25.2 \%$ of all respondents were found to be highly active. The percentage of all respondents with moderate level of physical activity was $35.9 \%$, while low level group amounted to $38.9 \%$. The percentages of men and women with high, moderate, and low levels of physical activity are presented in Fig. 1.

The analysis of the levels of physical activity in correlation to sex revealed differences only for males. Namely, men tended to state a high level of physical activity relatively less frequently

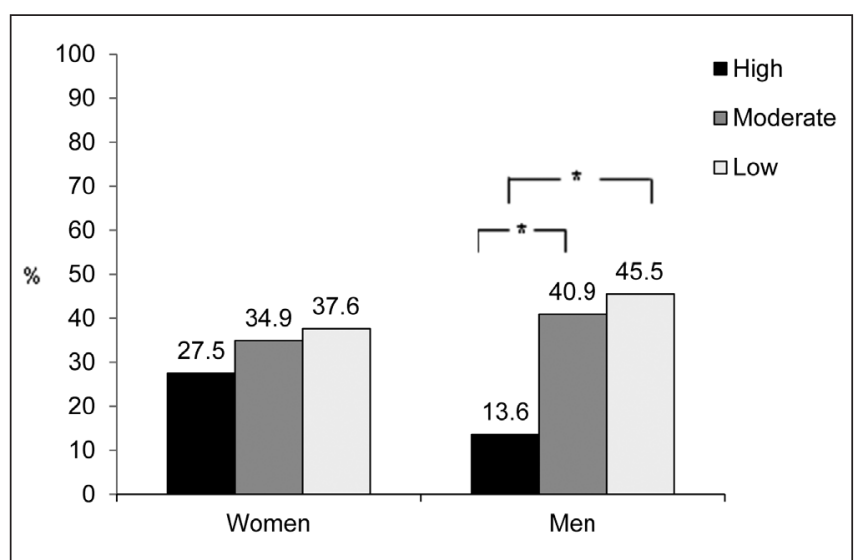

Fig. 1. The percentages of male and female students of the U3A with high, moderate, and low levels of physical activity.

* Significant differences $(p<0.05)$ between men: high vs. moderate and low levels of physical activity 
$(p<0.05)$ than moderate or low levels. There were no such differences among female respondents.

A short version of the WHOQOL-BREF questionnaire was used to assess the perceived quality of life. Detailed data is shown in Table 1.

Research results indicate that the level of physical activity (high, moderate or low) does not influence the general perception of quality of life (Table 2). The average assessment of general quality of life among the respondents displaying a high level of physical activity was $3.8 \pm 0.5$ points, while the results among students achieving moderate and low levels of physical activity were $3.6 \pm 0.5$ and $3.6 \pm 0.6$ points, respectively. On the other hand, the investigation revealed statistically significant differences $(p<0.05)$ in the respondents' assessment of their health condition depending on the presented level of physical activity. Namely, senior citizens characterized by a high level of physical activity (3.6 \pm 0.7$)$ assessed their health condition better than respondents reporting a moderate level of physical activity (3.2 \pm 0.8$)$.

The analysis of mean values for respective WHOQOL domains, related to the levels of physical activity of respondents, revealed significant differences within the sample (Table 2). Respondents with a high level of physical activity scored higher $(\mathrm{p}<0.05)$ in the psychological domain of the quality of life $(14.5 \pm 1.6)$ than students with moderate (13.4 \pm 1.8$)$ and low levels of physical activity $(13.0 \pm 1.6)$. Similarly, higher quality of life $(p<0.05)$ among the highly active respondents $(14.9 \pm 2.5)$, compared to the ones displaying a low level of physical activity, was also observed in the social domain. In the remaining two domains (physical and environmental), the quality of life was not correlated with levels of physical activity.

\section{DISCUSSION}

The influence of physical activity of senior citizens on their fitness and the phenomenon of so-called "successful ageing" are of great importance to their physical and psychological health. Research conducted in different countries shows that physical activity could improve the quality of life in older adults (26-28).

Despite the unquestionable benefits of physical exercise, Ferreira et al. (29) found that $87.2 \%$ of their respondents above 65 years of age did not undertake any physical activity in their free time, while $4.2 \%$ exercised less than 5 times a week, for less than 30 minutes. Nevertheless, it is worth noting that almost half of the respondents (49.6\%) were people aged 70-79, and 20.9\% were aged above 80 years. On the other hand, Brown et al. (30) indicate that even among fully fit individuals above 50 years of age, only $43.4 \%$ follow pro-health recommendations concerning physical activity.

A comparison with the presented U3A students indicates that the fraction of Polish senior citizens adhering to WHO recommendations is larger (61.1\%) than that of active (43.4\%) Columbians (30), or British (43.8\% of respondents aged 59-64) (31), but lower than the percentage of physically active respondents of Latvia (90.4\% aged 60-64) (32), or 60-70 years old Brazilians, investigated by Porto et al. (75.6\% of women and $76.1 \%$ of men) (33).

Sex-related analysis revealed that for both Polish (presented in the study, 62.4\%) and Vietnamese seniors (70.3\%), pro-health recommendations concerning physical activity were followed more often by women (34). Men less frequently achieved high and moderate levels of physical activity (Poles $54.5 \%$, Vietnamese $56.9 \%$ ). These findings are not confirmed by Brown et al. (30),

Table 1. Minimum, maximum, mean and standard deviations for respective WHOQOL-BREF domains among U3A students

\begin{tabular}{|l|c|c|c|}
\hline \multirow{2}{*}{} & \multicolumn{2}{|c|}{ Total N=131 } & $\bar{X} \pm$ SD \\
\cline { 2 - 4 } & $\min$ & 5.0 & $3.7 \pm 0.5$ \\
\hline General quality of life & 2.0 & 5.0 & $3.3 \pm 0.8$ \\
\hline Perceived health contidion & 2.0 & 18.3 & $12.8 \pm 1.5$ \\
\hline Physical domain & 8.0 & 18.0 & $13.5 \pm 1.8$ \\
\hline Psychological domain & 8.0 & 20.0 & $13.9 \pm 2.4$ \\
\hline Social domain & 9.3 & 19.0 & $13.8 \pm 1.8$ \\
\hline Environmental domain & 10.0 & & \\
\hline
\end{tabular}

Table 2. Mean values and standard deviations for respective WHOQOL-BREF domains in correlation with levels of physical activity among U3A students and statistically significant differences between them, calculated with the use of Kruskal-Wallis test

\begin{tabular}{|l|c|c|c|}
\hline \multirow{2}{*}{} & \multicolumn{2}{|c|}{ Level of physical activity } & Low \\
\cline { 2 - 4 } & High & Moderate & $\overline{\mathrm{X}} \pm$ SD \\
\cline { 2 - 4 } & $\overline{\mathrm{X}} \pm \mathrm{SD}$ & $\overline{\mathrm{X}} \pm \mathrm{SD}$ & $3.6 \pm 0.6$ \\
\hline General quality of life & $3.8 \pm 0.5$ & $3.6 \pm 0.5$ & $3.3 \pm 0.8$ \\
\hline Perceived health condition & $3.6 \pm 0.7^{c}$ & $3.2 \pm 0.8$ & $12.6 \pm 1.5$ \\
\hline Physical domain & $13.2 \pm 1.7$ & $12.8 \pm 1.4$ & $13.0 \pm 1.6$ \\
\hline Psychological domain & $14.5 \pm 1.6^{\mathrm{a}}$ & $13.4 \pm 1.8$ & $13.3 \pm 2.3$ \\
\hline Social domain & $14.9 \pm 2.5^{\mathrm{b}}$ & $13.8 \pm 2.3$ & $13.4 \pm 1.6$ \\
\hline Environmental domain & $14.3 \pm 1.7$ & $13.8 \pm 1.9$ & \\
\hline
\end{tabular}

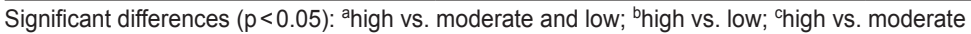


who claim that $46.5 \%$ of men respect the WHO recommendations, while only $41.1 \%$ of women do that. Different results obtained in this study may be caused by a small group of U3A students examined.

On the whole, international research proves that even relatively regular physical activity (moderate or vigorous physical activity) undertaken in the third age can have a positive influence on life satisfaction (30). Soyuer and Şenol (35) stated that old people (aged 65 and more) living in rest homes in Turkey showed that lower physical activity levels were connected with higher fatigue in older population. Nevertheless, scientists also point to the fact that the type of undertaken physical activity (at work, at home, or physical activity for transportation) can be of great importance in this respect. For example, it has been proven that there is a positive correlation only between physical activity for transportation and the general perception of quality of life (36). On the other hand, physical activity connected with transportation is inversely correlated with physical functioning of the HR-QOL domains whereas leisure-time physical activity was positively related to mental health of the HR-QOL domains (23). Research results indicate that also low and medium-high levels of walking for transportation was negatively correlated with mental and physical domains of the HR-QOL domains, but physical activity undertaken in leisure-time, in turn, has a positive influence on the perception of both domains (37). Moreover, Vuillemin et al. (38) claim that in case of physical activity undertaken in leisure time, the sense of quality of life (in all HR-QOL domains) is related to the intensity of physical activity. This means that all domains in SF-36 HRQOL and general health are assessed as better by seniors who exercise intensely.

In the cited studies $(23,36,37)$, levels of physical activity were assessed using a full version of IPAQ, while a short version was applied in this study. Nevertheless, Polish research results also confirm a positive correlation between the level of physical activity and health condition as perceived by respondents. The present study shows that highly active students of the University of the Third Age assessed their state of health better than respondents characterized by moderate level of physical activity. In turn, Omorou et al. (39) observed that the quality of life of males and females undertaking high and moderate physical activities is positively correlated with the better quality of life in the physical domain. Our results do not confirm such observations, because there were no statistically significant differences in the physical domains between seniors with high, moderate and low physical activity level. Omorou et al. (39) also observed correlations in psychological health but only in men undertaking high and moderate physical activity. In turn, we found differences in psychological domains between seniors with high, moderate and low physical activity level. Overall, Omorou et al. (39) noticed correlations in the domain of social relationships only in men with high physical activity, whereas our study showed that highly active seniors assessed better social domains than seniors with low level of physical activity. What is more, Pucci et al. (40) analysing correlations between the quality of life and physical activity undertaken in leisure time observed that walking for leisure was positively associated with psychological, physical and environment domains among women and only with environment and social domains among men.

The main limitation of this study is the small number of participants. Moreover, in our research, we analysed men and women together. The analysis could not be carried separately as the group of men consisted of a smaller number of participants.

Despite the use of different research methods, many results confirm that the level of physical activity recommended by WHO has a positive influence on the perceived quality of life. Possible differences are visible mostly in the assessment of different domains of life in relation to the presented levels of physical activity. The present study, using WHOQOL-BREF and a short version of IPAQ showed that highly active students of the University of the Third Age declare more often high quality of life in the psychological and social domains than other respondents.

On the basis of the above findings, it seems significant that strategies promoting the increase of physical activity among elderly people should emphasize its positive connection not only to better heath condition but also to improved quality of life.

\section{Conflict of Interests}

None declared

\section{REFERENCES}

1. Maniecka-Bryła I, Gajewska O, Burzyńska M, Bryła M. Factors associated with self-rated health (SRH) of a University of the Third Age (U3A) class participants. Arch Gerontol Geriatr. 2013 Sep-Oct;57(2):156-61.

2. Zielińska-Więczkowska H, Muszalik M, Kędziora-Kornatowska K. The analysis of aging and elderly age quality in empirical research: data based on University of the Third Age (U3A) students. Arch Gerontol Geriatr. 2012 Jul-Aug;55(1):195-9.

3. Zielińska-Więczkowska H, Kędziora-Kornatowska K, Ciemnoczołowski W. Evaluation of quality of life (QoL) of students of the University of Third Age (U3A) on the basis of socio-demographic factors and health status. Arch Gerontol Geriatr. 2011 Sep-Oct;53(2):e198-202.

4. Kimm H, Sull JW, Gombojav B, Yi SW, Ohrr H. Life satisfaction and mortality in elderly people: the Kangwha Cohort Study. BMC Public Health. 2012 Jan 19;12:54. doi: 10.1186/1471-2458-12-54.

5. Koivumaa-Honkanen H, Honkanen R, Viinamäki H, Heikkilä K, Kaprio J, Koskenvuo M. Life satisfaction and suicide: a 20-year follow-up study. Am J Psychiatry. 2001 Mar;158(3):433-9.

6. Diener E. Chan MY. Happy people live longer: subjective well-being contributes to health and longevity. Appl Psychol Health Well Being. 2011;3(1):1-43.

7. Veenhoven R. Healthy happiness: effects of happiness on physical health and the consequences for preventive health care. J Happiness Stud. 2008;9(3):449-69

8. Kao S, Lai KL, Lin HC, Lee HS, Wen HC. WHOQOL-BREF as predictors of mortality: a two-year follow-up study at veteran homes. Qual Life Res. 2005 Aug; 14(6):1443-54.

9. Koivumaa-Honkanen H, Honkanen R, Viinamäki H, Heikkilä K, Kaprio J, Koskenvuo M. Self-reported life satisfaction and 20-year mortality in healthy Finnish adults. Am J Epidemiol. 2000 Nov 15;152(10):983-91.

10. Borglin G, Jakobsson U, Edberg AK, Hallberg IR. Self-reported health complaints and their prediction of overall and health-related quality of life among elderly people. Int J Nurs Stud. 2005 Feb;42(2):147-58.

11. Arent SM, Landers DM, Etnier JL. The effects of exercise on mood in older adults: a meta-analytic review. J Aging Phys Act. 2000;8(4):407-30.

12. Menec VH. The relation between everyday activities and successful aging: a 6-year longitudinal study. J Gerontol B Psychol Sci Soc Sci. 2003 Mar;58(2):S74-82.

13. Shibata A, Oka K, Nakamura Y, Muraoka I. Recommended level of physical activity and health-related quality of life among Japanese adults. Health Qual Life Outcomes. 2007 Nov 28;5:64. doi: 10.1186/1477-75255-64.

14. Peddle CJ, Au HJ, Courneya KS. Associations between exercise, quality of life, and fatigue in colorectal cancer survivors. Dis Colon Rectum. 2008 Aug;51(8):1242-8. 
15. Lan TY, Chang HY, Tai TY. Relationship between components of leisure physical activity and mortality in Taiwanese older adults. Prev Med. 2006 Jul;43(1):36-41.

16. Fraga MJ, Cader SA, Ferreira MA, Giani TS, Dantas EH. Aerobic resistance, functional autonomy and quality of life (QoL) of elderly women impacted by a recreation and walking program. Arch Gerontol Geriatr. 2011 Jan-Feb;52(1):e40-3.

17. Giuli C, Papa R, Mocchegiani E, Marcellini F. Predictors of participation in physical activity for community-dwelling elderly Italians. Arch Gerontol Geriatr. 2012 Jan-Feb;54(1):50-4.

18. Fox KR. The influence of physical activity on mental well-being. Public Health Nutr. 1999 Sep;2(3A):411-8.

19. Chen LJ, Stevinson C, Ku PW, Chang YK, Chu DC. Relationships of leisure-time and non-leisure-time physical activity with depressive symptoms: a population-based study of Taiwanese older adults. Int J Behav Nutr Phys Act. 2012 Mar 14;9:28. doi: 10.1186/1479-5868-9-28.

20. Lee IM, Sesso HD, Oguma Y, Paffenbarger RS Jr. Relative intensity of physical activity and risk of coronary heart disease. Circulation. 2003 Mar 4;107(8):1110-6.

21. Rehn TA, Winett RA, Wisløff U, Rognmo O. Increasing physical activity of high intensity to reduce the prevalence of chronic diseases and improve public health. Open Cardiovasc Med J. 2013;7:1-8.

22. Bize R, Johnson JA, Plotnikoff RC. Physical activity level and healthrelated quality of life in the general adult population: a systematic review. Prev Med. 2007 Dec;45(6):401-15.

23. Jurakić D, Pedišić Z, Greblo Z. Physical activity in different domains and health-related quality of life: a population-based study. Qual Life Res. 2010 Nov;19(9):1303-9.

24. Biernat E, Stupnicki R, Gajewski AK. International Physical Activity Questionnaire (IPAQ) - Polish version. Wych Fiz Sport. 2007;51(1):4754. (In Polish.)

25. Wołowicka L, Jaracz K. Polish version of WHOQOL - WHOQOL 100 and WHOQOL-BREF. In: Wołowicka L, editor. Quality of life in medical sciences. Poznań: Dział Wydawnictw Uczelnianych Akademii Medycznej im. Karola Marcinkowskiego; 2001. p. 231-38. (In Polish.)

26. Ku PW, McKenna J, Fox KR. Dimensions of subjective well-being and effects of physical activity in Chinese older adults. J Aging Phys Act. 2007 Oct;15(4):382-97.

27. Acree LS, Longfors J, Fjeldstad AS, Fjeldstad C, Schank B, Nickel KJ, et al. Physical activity is related to quality of life in older adults. Health Qual Life Outcomes. 2006 Jun 30;4:37. doi:10.1186/1477-7525-4-37.

28. Rejeski WJ, Mihalko SL. Physical activity and quality of life in older adults. J Gerontol A Biol Sci Med Sci. 2001 Oct;56 Spec No 2:23-35.
29. Ferreira MT, Matsudo SM, Ribeiro MC, Ramos LR. Health-related factors correlate with behavior trends in physical activity level in old age: longitudinal results from a population in São Paulo, Brazil. BMC Public Health. 2010 Nov 10;10:690. doi: 10.1186/1471-2458-10-690.

30. Brown DR, Yore MM, Ham SA, Macera CA. Physical activity among adults $>$ or $=50$ yr with and without disabilities, BRFSS 2001. Med Sci Sports Exerc. 2005 Apr;37(4):620-9.

31. Choi M, Prieto-Merino D, Dale C, Nüesch E, Amuzu A, Bowling A, et al. Effect of changes in moderate or vigorous physical activity on changes in health-related quality of life of elderly British women over seven years. Qual Life Res. 2013 Oct;22(8):2011-20.

32. Kaupuzs A. A cross-sectional analysis of physical activity, psychological determinants and health related variables of Latvian older adults. Eur J Adapt Phys Activ. 2012;5(1):39-52.

33. Porto DB, Guedes DP, Fernandes RA, Reichert FF. Perceived quality of life and physical activity in Brazilian older adults. Motricidade. 2012;8(1):33-41.

34. Trinh OT, Nguyen ND, Dibley MJ, Phongsavan P, Bauman AE. The prevalence and correlates of physical inactivity among adults in Ho Chi Minh City. BMC Public Health. 2008 Jun 9;8:204. doi: 10.1186/14712458-8-204.

35. Soyuer F, Şenol V. Fatigue and physical activity levels of 65 and over older people living in rest home. Int J Gerontol. 2011;5(1):13-6.

36. Valadares AL, Carvalho ED, Costa-Paiva LH, Morais SS, Pinto-Neto AM. Association between different types of physical activities and quality of life in women aged 60 years or over. Rev Assoc Med Bras. 2011 Jul-Aug;57(4):450-5.

37. Gómez LF, Moreno J, Gómez OL, Carvajal R, Parra DC. Physical activity and health-related quality of life among adult women in Cali, Colombia: a cross-sectional study. Qual Life Res. 2013 Nov;22(9):2351-8.

38. Vuillemin A, Boini S, Bertrais S, Tessier S, Oppert JM, Hercberg S, et al. Leisure time physical activity and health-related quality of life. Prev Med. 2005 Aug;41(2):562-9.

39. Omorou YA, Erpelding ML, Escalon H, Vuillemin A. Contribution of taking part in sport to the association between physical activity and quality of life. Qual Life Res. 2013 Oct;22(8):2021-9.

40. Pucci G, Reis RS, Rech CR, Hallal PC. Quality of life and physical activity among adults: population-based study in Brazilian adults. Qual Life Res. 2012 Nov;21(9):1537-43.

Received October 17, 2014

Accepted in revised form June 1, 2015 